\title{
MANAGEMENT OF A CASE OF PRALLETHRIN POISONING-AN UNUSUAL AGENT FOR SUICIDAL INGESTION
}

\author{
Anbarasan Ardhanari ${ }^{1}$, Uma Srivastava ${ }^{2}$, Aditya kumar ${ }^{3}$, Surekha Saxena ${ }^{4}$ \\ Department of Anaesthesiology \& Critical Care, S.N.Medical College, Agra, Uttar Pradesh, \\ India ${ }^{1,2,3,4}$
}

*Corresponding author :sweetanbu2006@yahoo.co.in

Key words: Insecticide, poisoning

\begin{abstract}
Pyrethroids are widely used as commercial and domestic insecticides. Prallethrin is a type I pyrethroid compound and is used as a liquid mosquito repellant. Despite extensive use, there are relatively few reports of human pyrethroid poisoning. This report describes the management of a 20 year-old male patient who had consumed prallethrin.
\end{abstract}

Prallethrin is related to allethrin, a type I pyrethroid and is used as a liquid mosquito repellant. It has a basic cyclopropane carboxylic ester structure. Pyrethroids are widely used as commercial and domestic insecticides. It is available as a liquid mosquito repellant to be applied on the skin. Generally, pyrethroids are considered safe for human use because they have poor dermal absorption and rapid metabolism with little tissue accumulation, which have resulted in few reports of human toxicity. We are reporting a case of prallethrin poisoning due to deliberate self ingestion.

A young 20 year old male was admitted to our intensive care unit (ICU) in April 2010 with a history of vomiting, abdominal pain, dizziness, generalized convulsions and altered sensorium following intentional ingestion of approximately $25 \mathrm{ml}$ of liquid mosquito repellant, four hours before coming to hospital. Examination revealed a pulse rate of $121 /$ beats/min following a dose of atropine, blood pressure of $132 / 68 \mathrm{mmHg}$, respiratory rate $(\mathrm{RR})$ of 32 breaths $/ \mathrm{min}$, glasgow coma score (GCS)-E1, V1, M3, and bilateral crepitations. Pupils were semi-dilated with sluggish reaction to light, $\mathrm{SpO} 2$ was $75 \%$ on room air. The patient was intubated and ventilated. Blood samples were sent for serum electrolytes, haemogram, blood urea, serum creatinine and arterial blood gas (ABG) analysis. $\mathrm{ABG}$ analysis showed metabolic acidosis. Remaining blood investigations were in the normal range. Chest $\mathrm{X}$-Ray revealed features of interstitial pulmonary oedema. Mechanical ventilation with positive endexpiratory pressure was continued and $20 \mathrm{mg}$ of frusemide was given intravenously. The patient was given broad spectrum antibiotic coverage. The condition of the patient improved over 48 hours and was gradually weaned off the ventilator on the $4^{\text {th }}$ day. The patient complained of mild headache, dizziness and excessive fatigue which gradually improved. The patient was referred to a psychiatrist for counseling and was discharged on the $6^{\text {th }}$ day.

Discussion
Prallethrin is a synthetic insecticide
chemically related to pyrethroids.
Pyrethroids are used as insecticides. They
are about 2250 times more toxic to
insects than to mammals due to increased
sodium channel sensitivity, smaller body
size and lower body temperature in the 
former. Poor absorption through skin and rapid metabolism to inactive metabolites protects the mammals. The main toxic effects of pyrethroids are on voltagegated sodium channels by delaying their closure. At higher concentration pyrethroids can also act on GABA-gated chloride channels which causes seizure activity after toxicity ${ }^{1}$.

Occupational toxicity occurs through dermal absorption resulting in parasthesiae which recovers spontaneously in a few hours ${ }^{1}$. Ingestion of pyrethroid causes nausea, vomiting, abdominal pain, dizziness, headache, fatigue, palpitation, tightness in chest and blurring of vision. Coma, convulsions and pulmonary oedema are uncommon but can occur in severe poisoning ${ }^{2}$. These signs and symptoms of pyrethroid are very similar to those of organophosphate poisoning which made us use atropine prior to a diagnosis being made. The possibility that pyrethroids also induce hypersensitivity reactions, which may be fatal when the respiratory tract is involved, has been debated ${ }^{1}$.

There is no specific antidote for pyrethroid toxicity, therefore management is only symptomatic and supportive. Occupational toxicity resulting in parasthesia is treated by skin decontamination. Following ingestion of large amount of pyrethroids, gastrointestinal decontamination may be done if patients report to hospital within a few hours ${ }^{3,4}$.

This patient presented with convulsions, respiratory distress and altered sensorium. Endotracheal intubation and mechanical ventilation was instituted. Respiratory distress in this patient could be due to interstitial pulmonary oedema, developing as a result of a hypersentivity reaction. The pulmonary oedema may have been related also to the pyrethroid induced neuroexcitation and sympathetic surge due to release of norepinephrine ${ }^{5}$.

\section{Conclusion}

In our patient a clear history of liquid mosquito repellant ingestion was available. So we managed accordingly. Before we conclude, we would like to remind that the signs and symptoms of pyrethroid toxicity are similar to those of organophosphate poisoning. It is very essential to differentiate between the two, as few cases of death have been reported due to atropine toxicity ${ }^{2}$.

\section{References}

1. Bradberry SM, Cage SA, Proudfoot AT, Vale JA. Poisoning due to pyrethroids: Toxicology Review 2005;24:93-106.

2. He F, Wang S, Lui L, et al. Clinical manifestations and diagnosis of acute pyrethroid poisoning: Arch Toxicol 1989;63:54-8.

3. Flannigan SA, Tucker SB, Key MM, et al. Synthetic pyrethroid insecticides: A dermatological evaluation: $\mathrm{Br} \quad J$ Ind $\mathrm{Med}$ 1985;42:363-72.

4. Tucker SB, Flannigan SA and Ross CE. Inhibitions of cutaneous parasthesia resulting from synthetic pyrethroid exposure. Int $J$ Dermatol 1984;10:686-9.

5. Clark JM, Brooks MW. Neurotoxicology of pyrethroids: single or multiple mechanisms of action? Environ Toxicol Chem 1989;8:361-72. 